\title{
Blue-white screening liquid can eliminate false positives in blue-white colony screening
}

\author{
Y.S. Zhang1,2 \\ ${ }^{1}$ Department of Biotechnology, College of Chemistry and Biology Engineering, \\ University of Electronic Science and Technology of China Zhongshan Institute, \\ Zhongshan, China \\ ${ }^{2}$ Horticulture Section, School of Integrated Plant Science, Cornell University, \\ Ithaca, NY, USA \\ Corresponding author: Y. Zhang \\ E-mail: class2007ok@163.com
}

Genet. Mol. Res. 15 (2): gmr. 15027925

Received October 27, 2015

Accepted January 28, 2016

Published June 10, 2016

DOI http://dx.doi.org/10.4238/gmr.15027925

\begin{abstract}
Although blue-white screening based on $\alpha$-complementation has been widely used in the screening of genetically modified bacteria, only a single blue-white screening is typically not enough to eliminate false positives. Sometimes, a secondary blue-white screening for the target colonies is required. In this study, two methods were used to investigate the feasibility of secondary blue-white screening for target colonies on lysogeny broth (LB)-ampicillin agar plates. The first method consisted of covering the target colonies grown on LB-ampicillin plate medium with a sterilized filter paper soaked in a solution of $60 \mu \mathrm{L} 20 \mathrm{mg} / \mathrm{mL}$ X-gal and $8 \mu \mathrm{L} \mathrm{20 \%} \mathrm{IPTG.} \mathrm{The} \mathrm{second}$ method was that blue and white colonies were randomly selected from the blue-white screening plate medium and then re-streaked onto the blue-white screening medium. The colonies were then treated by two methods and incubated at $37^{\circ} \mathrm{C}$ for $12 \mathrm{~h}$. The results showed that some of the white colonies treated by the two methods showed results similar to the colonies grown on the blue-white screening medium. These results indicate that the target colonies grown on blue-white screening medium
\end{abstract}


can still be used to carry out a secondary blue-white screening. Thus, a blue-white screening liquid was successfully developed. Using the blue-white screening liquid, false positives can be eliminated directly based on the color of the target colonies. This will greatly improve the screening efficiency of positive clones and has important practical implications.

Key words: Blue-white screening liquid; Target colonies; False positive; Secondary blue-white screening;

\section{INTRODUCTION}

Gene cloning is regularly used in molecular biology research to investigate biological activity of candidate genes. Once a gene is isolated and cloned, it is possible to confirm the gene's activity by inserting it into another organism, closely or distantly related, to observe the phenotypic response. Conversely, silencing or knock-out experiments allow the researcher to observe the phenotype associated with the loss of the gene's expression in an organism. The process of gene cloning commonly involves several steps: candidate gene amplification, candidate gene ligation into a vector plasmid, transformation of the vector plasmid into bacteria, inoculation of selection media with transformed bacteria, and selection of positively transformed bacteria. Frequently, in preparation for cloning experimentation, the candidate gene is amplified using a polymerase chain reaction (PCR), in order to attain the necessary vector plasmid to candidate gene molar ratio of 1:3. Even though the higher concentration of the candidate gene would encourage a higher ligation rate, many vector plasmids still do not contain a ligated candidate gene insert. It is inevitable that many of these "empty" vector plasmids are transformed into the bacteria alongside the vectors carrying the candidate gene. Typically, the pooled bacteria will be spread onto selection media to screen for bacteria carrying the vector plasmid. A vector plasmid will also carry an antibiotic-resistance gene to promote a simpler selection of the bacteria that host the plasmid. Often ampicillin resistance genes are inserted into the plasmid for this purpose. Lysogeny broth (LB)-ampicillin culture medium is extensively used to improve the selection of transgenic bacteria following bacterial transformation experiments. When ampicillin is maintained at an acceptable concentration (50$100 \mathrm{mg} / \mathrm{mL}$ ) on LB-ampicillin medium, the use of ampicillin resistant vectors is very effective in the selection of positively transformed bacteria. However, these transformed bacteria need not carry the target gene insert. Indeed, many false positives arise in the application of LBampicillin medium screening of transformed bacteria. The presence of false positives growing on LB-ampicillin culture medium is a source of frustration to researchers and quickly reduces the efficiency of a cloning experiment. In order achieve a positive outcome, a researcher must overestimate the sample number of bacterial colonies, in order to find a positive transformation. This invariably causes further delay due to the need for subsequent validation.

In order to overcome the problem of false positives, blue-white screening based on $\alpha$-complementation can be used. $\alpha$-Complementation refers to the combination of two inactive segments ( $\mathrm{N}$-terminal segment and $\mathrm{C}$-terminal segment) of $\beta$-galactosidase, forming the intact, enzymatically active form of $\beta$-galactosidase in Escherichia coli (Zhou and Cao, 2010). Yet, even though blue-white screening has the potential to significantly improve selection efficiency, this technique sometimes results in reduced performance. White colonies 
are expected to carry the target gene, whereas blue colonies are not. Blue-white screening is centered on the complementary action of the $\alpha$-peptide ( $\mathrm{N}$-terminal with 11-41 residues deleted) with the $\mathrm{C}$-terminal, segment forming a functional $\beta$-galactosidase enzyme in $E$. coli. Formation of the intact and active $\beta$-galactosidase can be detected with the application of 5 -bromo-4-chloro-3-indolyl- $\beta$-D-galactopyranoside (X-gal). $\beta$-galactosidase cleaves $\mathrm{X}$-gal to form 5-bromo-4-chloro-indoxyl, which spontaneously dimerizes to form 5,5'-dibromo-4,4'dichloro-indigo, a blue pigment (Zhou and Cao, 2010). Enzymatic activity of $\beta$-galactosidase can be enhanced with the help of the inducer isopropyl $\beta$-D-1-thiogalactopyranoside (IPTG).

The nucleic region of the plasmid that expresses the $\alpha$-peptide, called lac $Z$, carries a multiple cloning site that can be cleaved with restriction enzymes for the insertion of the target gene. The presence of the foreign target gene inhibits the activation of $\beta$-galactosidase and $\mathrm{X}$-gal is not hydrolyzed, which results in reduced performance. Since blue-white screening can provide differentiation between the false positives (blue) and the positives (white), and the colonies with plasmid insertion from those without, it has the potential to greatly reduce the subsequent screening work. As a result of the greater potential for positive selection provided by this method, blue-white screening has been extensively used in transgenic research (Sherwood, 2003; Padmanabhan et al., 2011; Zhou and Cao, 2010; Li et al., 2015).

However, even after a number of white colonies have been selected from blue-white screening plate medium, positive clones cannot be obtained from expanding culture of liquid medium. This result can occur for many reasons. Likely reasons include that the number of individual colonies cultured on the plate medium is not high enough to display the blue strongly, that the competent cells were contaminated, or that the insertion of a foreign DNA fragment into the polycloning site of a plasmid almost invariably results in the production of an aminoterminal fragment incapable of $\alpha$-complementation (Sherwood, 2003). Yet another possibility could be that a few linearized vectors may be transformed into the bacteria, followed by the ligation of "repaired" ends, such that no LacZ gene is produced. These cells cannot convert $\mathrm{X}$-gal to form the blue pigmentation (Padmanabhan et al., 2011). Therefore, the false positives cultured in liquid medium cannot be directly identified, requiring further identification with subsequent indirect detection with techniques such as colony PCR screening.

Consequently, development of a high throughput method for screening blue-white colonies would have a positive impact on cloning efficiency. In this study, we explored the feasibility of a secondary screening method for improved selection of target colonies grown on LB-ampicillin medium. Based on our findings, a blue-white screening liquid was developed, through which the number of false positives can be markedly reduced based on their color. This is a simple, time-saving blue-white screening method.

\section{MATERIAL AND METHODS}

The preparation of competent DH5 $\alpha$ cells, LB-ampicillin culture medium, $20 \mathrm{mg} / \mathrm{mL}$ X-gal, and 20\% IPTG were performed following the Brook and Russell (2002) protocol. The target gene was amplified using conventional PCR methodology and purified by gel recovery. The target gene was ligated to a TA clone and transformed into E. coli. DH5 $\alpha$ transformed competent cells were smeared onto LB-ampicillin plate medium and the blue-white screening plate medium, respectively. Both kinds of plate media were incubated at $37^{\circ} \mathrm{C}$ for $12 \mathrm{~h}$ in a constant-temperature incubator.

The target colonies containing the target gene were selected from the ampicillin plate 
medium. A piece of sterilized filter paper was evenly soaked in $68 \mu \mathrm{L}$ solution containing $60 \mu \mathrm{L} \mathrm{X}$-gal $(20 \mathrm{mg} / \mathrm{mL})$ and $8 \mu \mathrm{L}$ IPTG $(20 \%)$. The moist filter paper was then placed on the target colony area, covering it. Meanwhile, blue and white colonies selected from the blue-white screening plate medium were re-streaked onto a new blue-white screening plate medium. Both treatment plates were incubated at $37^{\circ} \mathrm{C}$ in a constant-temperature incubator for $12 \mathrm{~h}$.

In addition, three kinds of the blue-white screening liquids containing different proportions of IPTG and X-gal were prepared. Three blue colonies and one white colony were inoculated in three kinds of blue-white screening liquid to determine which blue-white screening liquid contained the best proportions of IPTG and X-gal. Then, five white colonies selected from the blue-white screening plate medium were inoculated in the blue-white screening liquid with the best proportions of IPTG and X-gal. These samples were cultured on a $37^{\circ} \mathrm{C}, 200 \mathrm{rpm}$ shaking table for $12 \mathrm{~h}$. When the five colonies had been allowed to expand in this liquid, colony PCR screening and agarose gel electrophoresis were conducted.

\section{RESULTS}

\section{Blue-white screening results of colonies covered by sterilized filter paper}

Several blue colonies were observed on the sterilized filter paper on the ampicillin plate medium, as shown in Figure 1. This indicates that the colonies on the ampicillin plate medium can be used to perform the secondary blue-white screening.

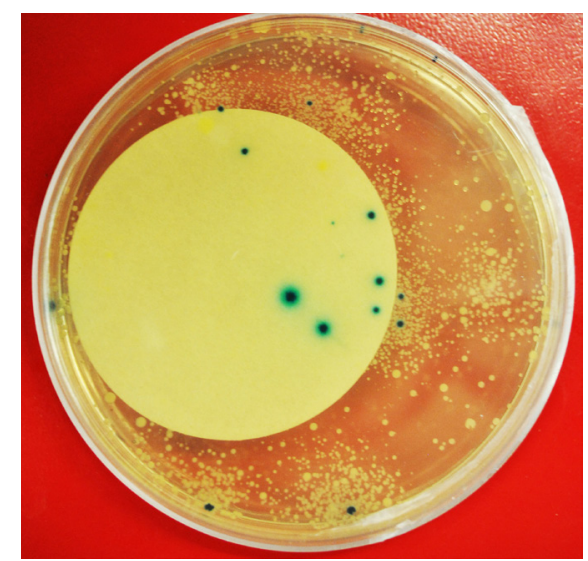

Figure 1. Color of colonies grown on LB ampicillin plate medium covered with moisturized sterilization filter paper. The sterilized filter paper was soaked using $68 \mu \mathrm{L}$ solution ( $60 \mu \mathrm{L} 20 \mathrm{mg} / \mathrm{mL}$ X-gal and $8 \mu \mathrm{L} 20 \%$ IPTG).

\section{Secondary blue-white scanning results on the blue-white screening plate medium}

The blue and white colonies randomly selected from the blue-white screening plate medium were vaccinated again onto the blue-white screening plate medium and cultured at $37^{\circ} \mathrm{C}$ in the constant temperature incubator for $12 \mathrm{~h}$. As shown in Figure 2, most of the blue colonies still displayed blue color on the blue-white screening plate medium, but several of the colonies were not obviously blue. This not only indicates that the result of one-time bluewhite screening is sometimes unreliable, but also shows that a secondary screening is useful. 


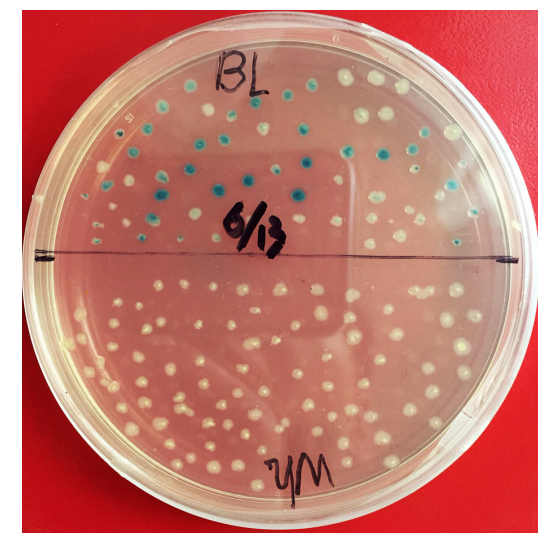

Figure 2. Secondary blue-white screening results. Blue (upper half) and white colonies (lower half) were selected from the blue-white screening plate medium and were inoculated again on the blue-white screening plate medium. Blue and white colonies were displayed after the plates had been incubated at $37^{\circ} \mathrm{C}$ for $12 \mathrm{~h}$.

\section{Different reagent proportions of the blue-white screening liquid and their respective color performance}

Blue-white screening liquid with different proportions of IPTG and X-gal showed differences in color after culturing the bacteria colonies. In this experiment, we used three bluewhite screening liquids containing different proportions of IPTG and X-gal (12/90, 16/120, and 8/60) in $5 \mathrm{~mL}$ LB-ampicillin liquid medium (Table 1). Three blue colonies and one white colony were cultured using the different screening liquids (Figure 3). Significant differences in color were shown between the blue (tubes 1, 3, and 4) and white (tube 2) colonies (Figure 3). The higher the proportion of IPTG to X-gal used, the darker the color of blue-white screening liquid is (tubes 1, 3, and 4; Figure 3). Considering the costs of the reagents used and the color, we propose that the best proportion of blue-white screening liquid is $5 \mathrm{~mL} \mathrm{LB}$-ampicillin liquid medium with a ratio of IPTG to X-gal of 12/90.

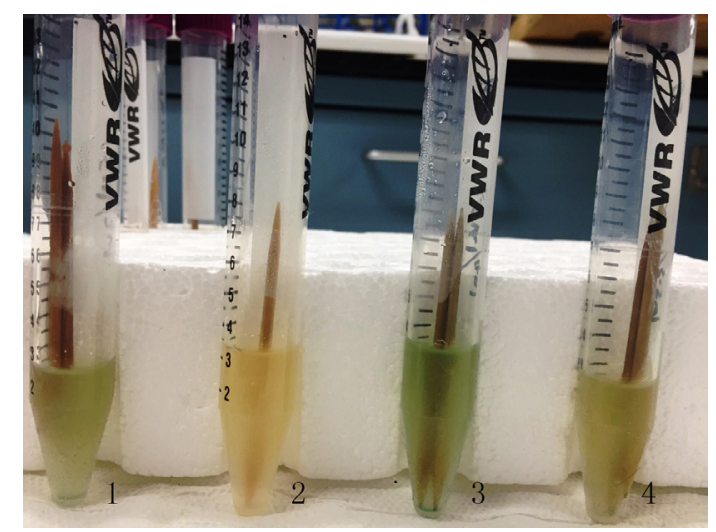

Figure 3. Color of screening liquid following colony culture in three different blue-white screening liquids differing in the proportions of IPTG and X-gal [12/90, 12/90 (white), 16/120, and 8/60]. 
Table 1. Three kinds of blue-white screening liquids differing in the proportions of IPTG and X-gal and the type of colony used.

\begin{tabular}{l|c|c|c|c}
\hline Reagents/tube No. & 1 & 2 & 3 & 4 \\
\hline IPTG $(\mu \mathrm{L})$ & 12 & 12 & 16 & 8 \\
\hline$X$-gal $(\mu \mathrm{L})$ & 90 & 90 & 120 & 60 \\
\hline Type of colony & Blue & White & Blue & Blue \\
\hline
\end{tabular}

\section{Blue-white screening liquid in combination with centrifugation for elimination of false positives and the evaluation of the screening results}

In order to eliminate false positives and improve the screening efficiency of positives, we developed a blue-white screening liquid. The liquid contained $5 \mathrm{~mL} \mathrm{LB}$-ampicillin liquid medium and $90 \mu \mathrm{L} 20 \mathrm{mg} / \mathrm{mL}$ X-gal and $12 \mu \mathrm{L} 20 \% \mathrm{IPTG}$. Five white colonies were randomly selected from the blue-white screening plate medium and were inoculated in the blue-white screening liquid for positive screening. Figure 4 (left) shows the color of the blue-white screening liquid in the five tubes after culturing of the target colonies at $37^{\circ} \mathrm{C}$ for $12 \mathrm{~h}$ before centrifugation. Following centrifugation, the color of the colonies differed greatly (as shown in Figure 4, right). Our results thus indicate that using the blue-white screening liquid in combination with centrifugation will have an improved effect in eliminating false positives, compared with using the method without subsequent centrifugation.
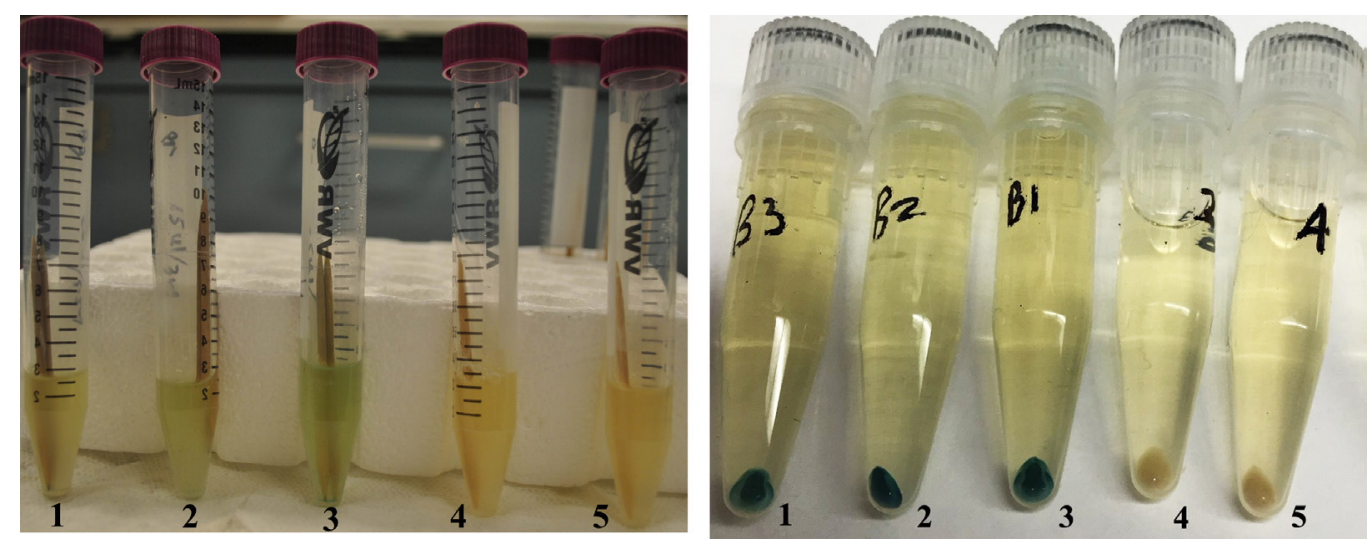

Figure 4. Colony color cultured in blue-white screening liquid before (left) and after (right) centrifugation.

However, we also found that there was a problem using blue-white screening liquid for selecting positive colony. In Figure 5, it can be observed that the first colony was a positive clone containing a foreign gene and its color should therefore be white. Oddly, this positive clone cultured in the blue-white liquid was a blue colony. This illustrates that it is necessary for some of the clones that screened positive using the blue-white screening liquid to be subjected to subsequent testing. By first screening the positive clones using the blue-white screening liquid, the number of subsequent positive testing colonies may be greatly reduced, thereby reducing the subsequent workload. 


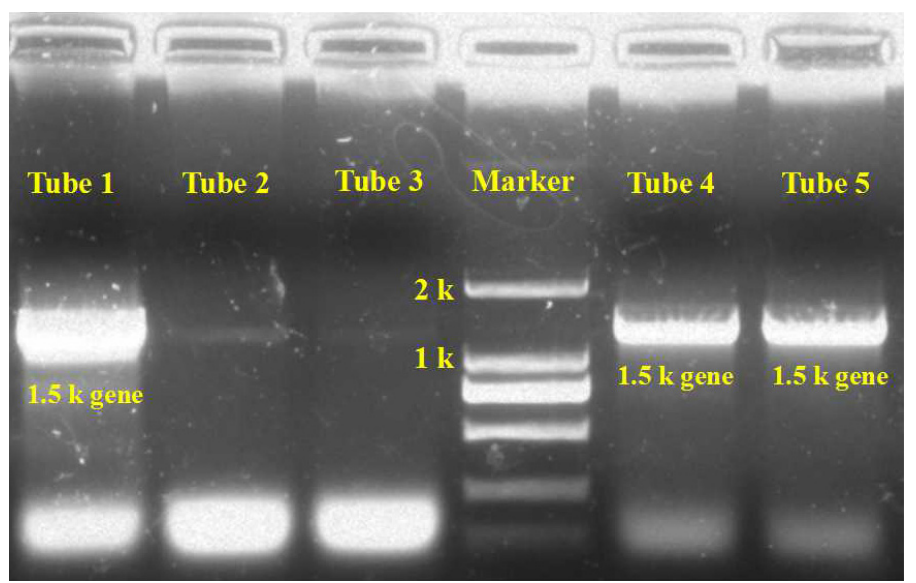

Figure 5. Results of agarose gel electrophoresis of the colonies corresponding to the colonies in Figure 4, following PCR.

\section{DISCUSSION}

\section{Colonies screened on LB-ampicillin plate medium can be used for subsequent blue-white screening}

The important conclusion in this study is that colonies screened on LB-ampicillin medium can still be used to perform blue-white screening. Based on our findings, we developed a successful blue-white screening liquid.

In general, bacteria grown on the blue-white screening plate medium are still in the logarithmic growth period and have high bacterial activity even after E. coli have been cultured for $12 \mathrm{~h}$. Therefore, the target colonies at this phase are suitable for secondary bluewhite screening. Our experimental results showed that the target colonies grown on the LBampicillin plate medium could still be used to carry out secondary blue-white screening. Our results not only provide strong evidence for the feasibility of secondary blue-white screening but have also laid the foundation for the direct development of the blue-white screening liquid.

\section{Color development of colonies grown in blue-white screening liquid is more sensitive than that grown on plates of blue-white screening medium}

This study shows that the color of colonies grown in the blue-white screening liquid is more sensitive than when grown in blue-white screening plate medium, since the liquid medium is more suitable for inoculum enlargement of target colony. There is limited nutrient around colonies grown on a plate of blue-white medium, which will lead to slow growth and a reduction the number of target colonies. Therefore, the color of blue or white colonies is sometimes not obvious and hard to identify, as shown in Figure 2. However, due to the mobility of the blue-white screening liquid, the local nutrient lack can be supplied or replenished from surrounding nutrients, which is conducive to the growth of a greater number of bacterial clones. Consequently, the color of target colonies grown in blue-white screening liquid is more obvious than that grown on a plate of blue-white screening medium. 


\section{Procedure for using the blue-white screening liquid}

Commonly, there are false positives in the blue-white screening plate medium. Since it is not possible to display colony color in the LB liquid culture medium, it is necessary to carry out further verification in the form of colony PCR and plasmid extraction, enzyme digestion, and so on. However, using blue-white screening liquid not only has the function of culturing the target colonies but can also make it possible to directly eliminate false positives based on their color. Hence, using blue-white screening liquid can reduce the workload and greatly improve the efficiency of positive selection. The improved blue-white colony screening procedure presented here includes: 1 ) the use of blue-white screening plate medium to screen white colonies, 2) selection of the target white colonies from blue-white screening plate medium have enlarged inoculum sizes in the blue-white screening liquid, 3) centrifugation of the solution of the target white colonies, and 4) the white colonies selected based on their color for subsequent experiments.

\section{Conflicts of interest}

The authors declare no conflict of interest.

\section{ACKNOWLEDGMENTS}

I am very grateful to Philip J. Hear from Plant Breeding and Genetics Section, School of Integrated Plant Science, Cornell University for writing the introduction of this paper. I would also like to thank the reviewers for their doing a lot of detailed modifications of the paper and for the language polish. Research supported by grants from the Fund of the Natural Science Foundation of Guangdong Province (\#8452840301001691), China.

\section{REFERENCES}

Brook SJ and Russell DW (2002). Molecular cloning: a laboratory manual. Science Press, Beijing

Li P, Li J, Guo Z, Tang W, et al. (2015). An efficient blue-white screening based gene inactivation system for Streptomyces. Appl. Microbiol. Biotechnol. 99: 1923-1933.http://dx.doi.org/10.1007/s00253-014-6369-0

Padmanabhan S, Banerjee S and Mandi N (2011). Screening of bacterial recombinants: strategies and preventing false positives. In: Molecular cloning - selected applications in medicine and biology (Gregory G, ed.). InTech Open Access Publisher, 3-20.

Sherwood AL (2003). Virtual elimination of false positives in blue-white colony screening. Biotechniques 34: 644-647.

Zhou Y and Cao J (2010). The research into mechanism of $\alpha$-complementation and blue-white screening. Biotechnol. Bull. 8: 218-221. 